Instituto Internacional de Investigación y Desarrollo Tecnológico Educativo INDTEC, C.A.

DOI: https://doi.org/10.29394/Scientific.issn.2542-2987.2018.3.10.8.154-174

OAI-PMH: http://www.indteca.com/ojs/index.php/Revista Scientific/oai

Artículo Original / Original Article

\title{
Uso de las Tecnologías de la Información y la Comunicación en la Educación Básica
}

Autores: Oscar Antonio Martínez Molina Universidad Nacional de Educación, UNAE oscar.martinez@unae.edu.ec Cuenca, Ecuador

\section{Resumen}

El propósito de este artículo es exponer los factores que inciden en el uso de las tecnologías de la información y la comunicación (TIC) por parte de los docentes de Educación Básica, lo que se ha convertido en un dilema profesional, debido a causas externas como la baja disponibilidad o ausencia de centros, software, equipos tecnológicos e Internet, los cuales de existir casi siempre están en manos de personas ajenas a la docencia, sumándose la escasez de cursos y programas nacionales de capacitación y entrenamiento en sistemas de informática y componentes de tecnología, y su insuficiente o nula inserción en el Currículo Nacional; esto repercute en el educador, quien presenta inexperiencia e incompletos conocimientos en el manejo y utilización de las TIC en el campo de la enseñanza y el aprendizaje de los menores, acarreándole situaciones de inseguridad, estrés y baja motivación, que, unidas muchas veces a la edad y resistencia al cambio, se reflejan en una educación que resulta no ser de alta calidad. Es una investigación cuantitativa, bibliográfica, descriptiva y estudio de caso; con una población de 26 docentes, donde se concluye la necesidad de superar tales deficiencias en pro de un óptimo proceso educativo para quienes inician la educación formal. básica.

Palabras clave: tecnología de la información; TIC; docente; educación 


\title{
Use of Information and Communication Technologies in Basic Education
}

\begin{abstract}
The purpose of this article is to expose the factors that affect the use of information and communication technologies (ICT) by basic education teachers, which has become a professional dilemma, due to external causes such as the low availability or absence of centers, software, technological equipment and the Internet, which almost always exist in the hands of people outside of teaching, adding to the scarcity of courses and national training and training programs in computer systems and components of technology, and its insufficient or no insertion in the National Curriculum; This affects the educator, who has inexperience and incomplete knowledge in the use and management of ICT in the field of teaching and learning of minors, leading to situations of insecurity, stress and low motivation, which, together many times to age and resistance to change, are reflected in an education that turns out not to be of high quality. It is a quantitative, bibliographic, descriptive and case study; with a population of 26 teachers, which concludes the need to overcome such deficiencies in favor of an optimal educational process for those who start formal education.
\end{abstract}

Keywords: information technology; ICT; teacher; basic education.

Date Received: 18-05-2018

Date Acceptance: 20-09-2018 


\section{Introducción}

Hoy en día, a nivel mundial, la:

emergente sociedad de la información, impulsada por un vertiginoso avance científico, en un marco socioeconómico neoliberal y globalizador, sustentado por el uso generalizado de las potentes y versátiles tecnologías de la información y la comunicación (TIC), conlleva a grandes cambios que alcanzan todos los ámbitos de la actividad humana (Marqués, 2006, pág. $3)$.

De tal manera que los efectos de las TIC, se manifiestan de manera muy especial, entre otros, en las actividades laborales, familiares y en el mundo educativo; aclarando que en éste último, todo puede ser atendido por las tecnologías, desde la razón de ser de la escuela y demás instituciones educativas, hasta la formación básica que precisan las personas, la forma de enseñar y de aprender, los conocimientos, las motivaciones, las destrezas y los medios utilizados para ello, puesto que ellas constituyen una vía para acceder y estar a la par de un gran cúmulo de información, sobre todo a la velocidad vertiginosa con la cual se difunde.

Tales tecnologías en el campo educativo, muy especialmente en Educación Básica, objeto de este artículo, permiten que la información llegue y se difunda, haciendo del Internet un medio informativo digital, que propicia que se haga menos tedioso, más rápido y motivante el aprendizaje, ya que los niños, en esta etapa, podrán disponer de una serie de herramientas informáticas, que harán de su aprendizaje algo rápido y novedoso, en pro de su desarrollo personal.

Las TIC son un medio de expresión para la creación, a través del uso de procesadores de texto y gráficos, editores de páginas web, presentaciones multimedia, cámaras de vídeo, entre otros; son además un canal de comunicación interpersonal, que contribuye con el trabajo colaborativo y con el intercambio de información e ideas, con uso de e-mail, foros telemáticos. 
Las TIC, deben ser conocidas y adoptadas por los educadores -los del nivel básico no quedan exentos- ya que ellas pueden contribuir dentro del campo pedagógico del niño, al desarrollo psicomotor, la adquisición de habilidades cognitivas, afianzar la identidad y la autonomía personal, el perfeccionamiento del lenguaje y la comunicación, crear pautas elementales de convivencia y relación social, y facilitar el descubrimiento del entorno inmediato.

Sin embargo, de lo señalado, no todos los docentes poseen un dominio de las TIC, y menos aún se proponen adquirir los conocimientos necesarios sobre las mismas, para utilizarlas en beneficio de los educandos. Es por esto que este artículo tiene como propósito exponer los factores que inciden en el uso de las tecnologías de la información y la comunicación (TIC) por parte de los docentes de Educación Básica, que inciden, niegan y retrasan el uso de estas tecnologías. Entre estos se destacan la inexistencia y déficit de software, equipos de computación (hardware) e Internet; en muchas instituciones educativas, además de falta de programas de capacitación y actualización del maestro, lo que repercute en el propio profesional (factores internos) es el desconocimiento del tema, apatía, baja motivación e incapacidad para usar las TIC como estrategias didácticas al servicio de la educación, pudiendo influir además la edad y la resistencia al cambio que posea el educador, todo lo que incidirá negativamente en el aprendizaje, crecimiento y desarrollo integral de los infantes.

\section{Estado Del Arte}

\subsection{Un espacio para las tecnologías de la información y la comunicación TIC, los docentes y la Educación Básica en Ecuador}

De acuerdo con (Buela-Casal y Castro, 2009, pág. 3):

las tecnologías de la información y la comunicación (TIC) se definen como aquellas herramientas que se encargan del 
estudio, desarrollo, implementación, procesamiento, almacenamiento y distribución de todo tipo de información, mediante la utilización de hardware y software como medios de sistemas informáticos.

Ellas hacen referencia a su utilización con diferentes finalidades, entre otras, la organización y gestión empresarial, así como la formación educativa, la adquisición, reposición y cambio de conocimientos y saberes, en todos los niveles de la educación, informal, no formal y formal, esta última referida a las universidades y otros recintos y niveles de formación del educando.

Para (Castro, Guzmán y Casado, 2007, pág. 230): las TIC dentro de la educación, se asumen como un eje integrador, sirviendo como recursos para:

construir ambientes de aprendizaje que permitan el desarrollo de estrategias, actividades, contenidos y materiales didácticos; coadyuvar en el desarrollo de proyectos pedagógicos, basados en intencionalidades educativas como aprender a crear, aprender a convivir y participar, aprender a valorar y aprender a reflexionar; fomentar valores ciudadanos, que conlleven al desenvolvimiento en entornos basados en tecnologías; impulsan la investigación desde la praxis pedagógica; desarrollan recursos didácticos que potencian el aprendizaje significativo y contextualizado.

Para la Educación Básica ecuatoriana, la función principal de la educación es conservar y transmitir las verdades sistemáticas y morales que son tan esenciales para el progreso de la sociedad, porque al final el objetivo de enseñar no es solamente el de comunicar conocimiento a quienes carecen de este, sino que el estudiante se adueñe del conocimiento aprendido por el docente.

En este nivel, las TIC juegan un rol fundamental en la formación de los menores, ya que el uso de estos ambientes de aprendizaje tecnológicos, acompañados de sus docentes, facilita y permite una interacción eficiente y productiva. Es importantes también para la elaboración de contenidos, la 
divulgación de trabajos y tareas, la resolución de problemas y la toma de decisiones, en medio de un clima colaborativo, cooperativo, enriquecedor y motivante que invita a seguir adelante en forma individual y grupal, en beneficio del desarrollo de los infantes.

Por otro lado los contenidos y las estrategias administrados con el uso de las TIC, permiten al docente junto a sus pequeños educandos, socializar y adaptarse al tiempo y a las posibilidades de cada quien; donde el computador, la Internet, los simuladores de situaciones y sitios reales, y los juegos electrónicos educativos (de memoria, rompecabezas, completación, pareo, entre otros), son vías para el aprendizaje y el desarrollo del ser, enmarcados en pilares que facilitan el desarrollo del pensamiento lógico, la argumentación, la meta-cognición, la descripción, clasificación, agrupación, seriación, comparación, así como la ubicación espacial y temporal, usando y valiéndose de medios que les resultan a los niños herramientas para el trabajo y la formación, sumamente amenos y recreativos.

Se agrega que el computador para los niños puede tornarse una vía fundamental para provocar nuevas interacciones, interrogarlos, proponerles nuevos problemas para mejorar y expandir las experiencias para resolverlos, usando ciertos programas computacionales lúdicos que permiten en conjunto, la formación, la ejercitación y la diversión.

Después de lo señalado, hay que indicar que en el país se están presentado serias situaciones problemáticas, asociadas al desconocimiento y puesta en práctica de TIC en los diferentes niveles educativos, aunque en este artículo nos centraremos en el nivel de Educación Básica.

\subsection{Problemas generados por desconocimiento y ausencia de la utilización de las (TIC) por parte de los docentes de Educación Básica}

(Vaillant, 2014, pág. 1130), considera que:

Las TIC son hoy una fuente de influencia y generación de 
modelos, patrones sociales y valores que nos hacen necesariamente repensar los procesos de aprendizaje y los procesos de enseñanza. $Y$ es en ese contexto que la investigación realizada indaga acerca de cómo deben aprender y cómo deberían aprender los docentes en el contexto de la sociedad de la información, del conocimiento y de las tecnologías.

Sin embargo, muchos de los educadores confrontan ciertas problemáticas referentes al caso:

- La formación resulta ser un proceso no necesariamente permanente, más bien alejado de un modelo de socialización, donde poco se incorporan conocimientos y prácticas que ayuden al análisis de diversas situaciones relacionadas con la institución, los estudiantes, los saberes y la toma de decisiones; en este sentido, el desconocimiento y la ausencia del uso TIC, no promueve procesos integrados que conlleven a la mejora de los métodos didácticos aplicados en las aulas, por lo tanto no se consigue del todo un desarrollo significativo que genere innovación educativa.

- Un desarrollo profesional alejado de la comprensión y utilización de las TIC en las clases, impide que la tecnología se integre y forme parte de los nuevos enfoques educativos, desmejorándose el enriquecimiento del aprendizaje de los niños involucrados, lo que podría generar un fuerte impacto negativo en su desarrollo y en su futuro desenvolvimiento en la formación a niveles superiores, en la comunidad, en el mundo laboral y en la sociedad en general, ya que ese menor se va a encontrar en una posición deficitaria frente a otros que sí han disfrutado de los espacios tecnológicos.

- Se puede decir que los profesores no cuentan con una serie de competencias de cara a la utilización de las TIC, en cuanto a los contenidos y área de trabajo específicas se refiere; hecho que puede manifestarse negativamente no sólo en su labor formadora sino que además podría repercutir en sus planificaciones de aula, en el dominio para la inserción de 
nuevas técnicas, estrategias y medios para la educación, en cualquier espacio y tiempo que combine la presencialidad y el aprendizaje grupal, con la formación a distancia o individualizada; agregándose la incapacidad para elaborar y seleccionar materiales y apoyos didácticos novedosos que faciliten y despierten en los niños el interés y la motivación para adquirir y reforzar conocimientos.

- Se agrega, de acuerdo con los datos obtenidos en la encuesta aplicada a los 26 docentes, que apenas el treinta por ciento $(30 \%)$ de los docentes involucrados, utilizan las TIC como un instrumento de innovación educativa, y aunque se cuente con cetros dotados de tecnología de punta, éstos no son usados en beneficio del proceso de enseñanza y aprendizaje.

- Además, sólo un sesenta por ciento (60\%), conoce las tecnologías y no en su totalidad; básicamente de la Internet, se valen de Facebook y del correo electrónico, en algunos casos se usan para los trabajos administrativos, y muy poco como herramientas didácticas de apoyo a la formación de los menores, para preparar las clases $(5 \%)$, o para la programación de objetivos, contenidos y actividades relacionadas a las TIC.

- Finalmente se expone, que tan solo un diez por ciento (10\%), usa las TIC, para sus actividades fuera del aula; aclarando que muchos de los conocimientos sobre las tecnologías los han adquirido por ensayo y error, por interés personal, a través de la observación de videos, y en muy pocos casos con cursos particulares, que sólo permiten el manejo de lo muy básico; además de la presencia de altas ocupaciones laborales, que se traducen en falta de tiempo para estudiar, así como bajo interés, o ausencia de oportunidades y dinero para capacitarse en relación a ellas.

Se destaca que los resultados demuestran que un bajo porcentaje de los docentes involucrados hacen honor a las tecnologías, tanto en su vida cotidiana, como en sus diferentes ambientes de trabajo pedagógicos. 


\subsection{Factores que inciden en el uso de las (TIC), por parte de los docentes de Educación Básica}

En función del punto anterior, seguidamente se presentan una serie de factores considerados de naturaleza externa e interna, que están incidiendo en el docente y el uso de la TIC en el nivel de Educación Básica, siendo éstos los siguientes:

\subsubsection{Factores externos}

Son considerados externos, aquellos factores que no están asociados, ni son responsabilidad directa del docente, pero que de alguna manera inciden en el uso de las TIC por parte de los profesionales de la Educación Básica en el país, encontrándose los mencionados seguidamente:

- Escases o ausencia de centros informáticos y bibliotecas virtuales, encargadas de suministrar información válida y actualizada a quienes acuden a ellas, como una vía para alcanzar la que puede llamarse alfabetización digital. Aquí entendemos biblioteca digital como "un sistema de tratamiento técnico, acceso y transferencia de información digital, estructurado alrededor del ciclo de vida de una colección de documentos digitales, sobre los cuales se ofrecen servicios interactivos de valor añadido para el usuario final" (Tramullas, 2002, pág. 8). Encontramos también ausencia o escases de computadores para alumnos y docentes, las cuales, de existir, casi siempre está en manos y son responsabilidad de un personal técnico, que siempre va a ser un profesional ajeno a la docencia, sobre todo en el nivel Inicial, donde se debe contar con equipos para satisfacer la curiosidad, aumentar el conocimiento y favorecer el aprendizaje significativo de quienes inician la educación formal. En muchos casos, además de no contarse con la conexión de Internet, los docentes no tienen apoyo para el uso de las TIC, no es clara la existencia de un plan de mantenimiento, actualización y seguridad para el hardware y el software, y menos aún se conoce si existen actividades tanto 
para alumnos como para profesores.

- Finalmente tenemos que citar la escasez de cursos y programas nacionales de capacitación y entrenamiento en sistemas de informática y componentes de tecnología, y su insuficiente o nula inserción en el Currículo Nacional. Al respecto, (Álvarez, Cuéllar, López, Adrada, Anguiano, Bueno, Comas y Gómez, 2011, pág. 10), manifiestan la necesidad de que: "los docentes se apropien de las TIC y que las incluyan en sus actividades, así como que generen con estas comunidades docentes que permitan el libre acceso a recursos educativos y tecnológicos de calidad en todas las escuelas".

\subsubsection{Factores internos}

Se entiende por factores internos, los que están directamente asociados al docente, dependen y son su responsabilidad, en su relación con las TIC en el nivel de Educación Básica, considerándose los siguientes:

- Inexperiencia, conocimientos incompletos y deficitarios en cuanto al manejo y utilización de las TIC en el campo de la enseñanza y el aprendizaje de los menores; en este sentido (García, 2004, págs. 513-515), sostiene que:

hay necesidades de formación del profesorado y carencias técnicas estructurales para dicha capacitación; donde las primeras hacen referencia a una serie de acciones encaminadas a la instrucción, o bien a aprender las habilidades básicas, instrumentales vinculadas a las nuevas tecnologías; es decir, se refiere al conocimiento que requieren y su utilización en los niños, en los entornos relacionados con el computador y el Internet. En cuanto a la segunda, es fundamental disponer de medios (computador, internet, otros), y materiales didácticos (software), para apoyar ese aprendizaje, que luego debe ser utilizado en la formación y crecimiento integral de los infantes.

- El desconocimiento y total abandono de las TIC puede acarrear en el docente situaciones de inseguridad, estrés y baja motivación para enfrentarse a los niños, quienes podrían tener un mejor y mayor manejo del computador 
que su mismo profesor; para este caso, Hüther (2012, págs. 47-48), expone que:

se puede presentar en el educador una reacción fisiológica de su organismo, donde entran en juego diversos mecanismos de defensa, para afrontar situaciones asociadas a las TIC, que se perciben como amenazantes o de alta demanda, generándole reacción a tal desafío que prefiere no abordar.

- Otros factores que puede influir en el uso de la TIC por parte del docente de Educación Básica son la actitud y la resistencia al cambio; en este último caso, (Mejía, Silva, Villarreal, Suarez y Villamizar, 2018, pág. 54), expresan que: "no querer, no poder o no conocer algo lo suficiente o en qué consiste, en este caso las TIC por el educador, son razones que tienden a demorar dicho cambio, lo que es percibido como cierta forma de resistencia". Esta ignorancia es generalmente ocasionada por falta de comunicación sobre el proyecto a modificar, el impacto que pueda tener en términos personales, además de poseer una visión demasiado parcializada del mismo; en los tres casos se hace evidente la ausencia de suficiente información sobre el tema, lo que hace que el adulto sienta incapacidad y dificultades para el trabajo en equipo junto a sus pequeños educandos, además del despertar de sentimientos negativos, como el desacuerdo, la incertidumbre, el temor por falta de confianza en sus resultados, y hasta la pérdida de identidad.

\section{Metodología}

Este trabajo responde al tipo de investigación cuantitativa, descriptiva y transversal Cuantitativo, ya que como indican (Taylor y Bogdan, 2000, pág. 5):

se caracteriza por buscar los hechos o causas de los fenómenos con independencia de los estados subjetivos de las personas que se encuentran cercanas, obteniéndose una visión de la realidad, como un espacio estable y estructurado, donde se relacionan los factores causales que afectan una determinada situación. 
Es de carácter descriptivo porque estudia, caracteriza y describe un fenómeno o situación concreta, indicando sus rasgos más peculiares o diferenciadores; también es de carácter transversal porque mide un hecho en un solo momento temporal.

\subsection{Población y muestra}

La población está conformada por los docentes de las escuelas de Educación Básica "Ignacio Andrade Arízaga" y "Ángela Rodríguez". En vista del tamaño de la población se ha trabajado con la totalidad es decir 26 , distribuidos de la siguiente manera:

Tabla 1. Escuelas objeto de estudio

\begin{tabular}{|l|c|c|}
\hline \multicolumn{1}{|c|}{ Escuela Básica } & Docentes & \multicolumn{1}{c|}{ Género } \\
\hline $\begin{array}{l}\text { Escuela de Educación Básica } \\
\text { Ignacio Andrade Arízaga }\end{array}$ & 13 & $\begin{array}{l}\text { Femenino: } 9 \\
\text { Masculino: } 4\end{array}$ \\
\hline $\begin{array}{l}\text { Escuela de Educación Básica } \\
\text { Ángela Rodríguez }\end{array}$ & 13 & $\begin{array}{l}\text { Femenino: } 7 \\
\text { Masculino: } 6\end{array}$ \\
\hline \multicolumn{1}{|c|}{ Total } & 26 & 26 \\
\hline
\end{tabular}

Fuente: Estadísticas de las escuelas (2017); El Autor (2018).

\subsection{Elaboración del instrumento}

La encuesta aplicada a los docentes estuvo estructurada por 16 preguntas que conformaron los 6 indicadores de la investigación. El cual, se validó a través de 3 expertos en tecnología, información y comunicación en el área educativa.

Para la aplicación del instrumento se empleó una herramienta digital y pagada, disponible en la Internet, denominada SurveyMonkey, que es una herramienta que posibilita la elaboración de preguntas abiertas y cerradas, y facilita la recogida de la información y su análisis con estadística descriptiva e inferencial, así como la relación de respuestas abiertas para el análisis de sus tendencias principales. 


\section{Análisis e Interpretación de Resultados}

A continuación, se presenta el análisis detallado y la interpretación de los resultados de la investigación, contextualizándolos y fundamentándolos con los datos y la bibliografía. Primero se presenta la información general sobre lo que se pudo observar y conocer en las escuelas visitadas, alguna cuantitativa, con el propósito de contextualizar los demás resultados.

Las TIC en las escuelas objeto de estudio no son algo nuevo, ya que las mismas tienen varios años aplicándose, principalmente por computadoras y actualmente con el uso de internet, por parte de los profesores. Sin embargo, no todos los docentes han estado en contacto con ellas, ya que la mayoría de las veces es el docente de computación el que mantiene una frecuente usabilidad de estos recursos. La pregunta que se plantea sería, si las TIC en las escuelas no son algo nuevo, ¿por qué para los docentes es todavía difícil incorporarlas a su práctica docente?

En los laboratorios de computación de ambas escuelas, el número de estudiantes que disponen de computadoras varia. El número de alumnos por escuela va desde 249 hasta 118, y el número de alumnos por computadora va desde 2.4 hasta 15. Ambas escuelas cuentan con sala de computación.

Finalmente, es de señalar que los estudiantes de ambas escuelas, por estar ubicadas en zonas rurales tienen acceso a computadoras e internet fuera de las escuelas.

Bajo esta situación planteada, debe tomarse en consideración que los estudiantes están familiarizados en cierta medida con los periféricos de hardware, por lo que se debe hacer hincapié más sobre el proceso enseñanza aprendizaje para que los estudiantes aprendan con ella.

Esta afirmación precisa que la mayoría de los estudiantes ya están alfabetizados en el uso de la tecnopedagogía para aprender de forma lúdica y diferente. 
Los indicadores que se plasman en el análisis de la encuesta son:

- Pertinencia del uso de la tecnología educativa.

- Elaboración de materiales educativos en soporte digital.

- Materiales audiovisuales.

- Materiales en línea, Internet.

- Redes sociales.

- Plataformas educativas (edmodo, moodle).

Tabla 2. Indicador: Pertinencia del uso de la tecnología educativa.

\begin{tabular}{|l|c|c|}
\hline \multicolumn{1}{|c|}{$\begin{array}{c}\text { Indicador: Pertinencia del uso de la } \\
\text { tecnología educativa }\end{array}$} & Selecciones & \% del total \\
\hline La evito constantemente & 2 & 7.69 \\
\hline No la evito, pero me desagrada & 4 & 15.38 \\
\hline Participo como parte de mi formación & 20 & 76.92 \\
\cline { 1 - 1 } Despierta mi motivación & & \\
\hline Disfruto realizarla & & \\
\cline { 1 - 1 } Está entre lo que más disfruto de la carrera & & \\
\hline
\end{tabular}

Fuente: Datos obtenidos del cuestionario (2017); El Autor (2018).

Los docentes objeto de estudio, en su inmensa mayoría, tienen experiencia en el empleo de las TIC en actividades recreativas, no tanto en actividades académicas. Dos docentes indican que evitan o que les desagrada el uso de la tecnología educativa no, otros cuatro la emplean para su formación, pero no parece que sea de su agrado, sin embargo, la tendencia predominante indica casi el $77 \%$ de los docentes asumen con responsabilidad su formación desde esta modalidad. 
Tabla 3. Indicador: Elaboración de materiales educativos en soporte digital

\begin{tabular}{|l|c|c|}
\hline $\begin{array}{c}\text { Indicador: Elaboración de materiales } \\
\text { educativos en soporte digital }\end{array}$ & Selecciones & $\begin{array}{c}\% \text { del } \\
\text { total }\end{array}$ \\
\cline { 1 - 1 } No se aprecia & 2 & 7.69 \\
\cline { 1 - 1 } Se aprecia muy poco & 5 & 19.23 \\
\cline { 1 - 2 } Se aprecia & 19 & 73.07 \\
\cline { 1 - 2 } Se aprecia bien & & \\
\cline { 1 - 2 } Es muy evidente la relación & &
\end{tabular}

Fuente: Datos obtenidos del cuestionario (2017); El Autor (2018).

Los materiales científicos en soporte digital constituyen fuente esencial para el proceso de apropiación del conocimiento relacionado con las materias con un reconocimiento del $92.30 \%$.

Tabla 4. Indicador: Materiales audiovisuales

\begin{tabular}{|l|c|c|}
\hline \multicolumn{1}{|c|}{ Indicador: Materiales audiovisuales } & Selecciones & $\begin{array}{c}\% \text { del } \\
\text { total }\end{array}$ \\
\cline { 1 - 1 } No se aprecia & 3 & 11.53 \\
\cline { 1 - 1 } Se aprecia muy poco & 6 & 23.07 \\
\cline { 1 - 1 } Se aprecia & 17 & 65.38 \\
\cline { 1 - 2 } Se aprecia bien & & \\
\cline { 1 - 2 } Es muy evidente la relación & &
\end{tabular}

Fuente: Datos obtenidos del cuestionario (2017); El Autor (2018).

De la misma forma los materiales audiovisuales tienen un lugar esencial en el proceso de formación y autoformación de los docentes, tal y como se exige en el modelo curricular de la actualización curricular de Educación Básica. El $88.45 \%$ de los docentes aprecian el valor de su empleo. 
Tabla 5. Indicador: Materiales en línea

\begin{tabular}{|l|c|c|}
\hline \multicolumn{1}{|c|}{ Indicador: Materiales en línea } & Selecciones & $\begin{array}{c}\% \text { del } \\
\text { total }\end{array}$ \\
\cline { 1 - 1 } No se aprecia & 3 & 11.53 \\
\cline { 1 - 1 } Se aprecia muy poco & 6 & 23.07 \\
\cline { 1 - 1 } Se aprecia & 17 & 65.38 \\
\cline { 1 - 2 } Se aprecia bien & & \\
\cline { 1 - 2 } Es muy evidente la relación & &
\end{tabular}

Fuente: Datos obtenidos del cuestionario (2017); El Autor (2018).

Relacionado con el tema de los materiales en línea el porcentaje de docentes que aprecian su empleo se encuentra sobre el $88 \%$. Es una cifra significativa, sin embargo, es uno de los aspectos donde se podría perfeccionar su empleo.

Tabla 6. Indicador: Internet

\begin{tabular}{|l|c|c|}
\hline \multicolumn{1}{|c|}{ Indicador: Internet } & Selecciones & $\begin{array}{c}\% \text { del } \\
\text { total }\end{array}$ \\
\cline { 1 - 1 } No se aprecia & 3 & 11.53 \\
\hline Se aprecia muy poco & 5 & 19.23 \\
\hline Se aprecia & 18 & 69.23 \\
\hline Se aprecia bien & & \\
\hline Es muy evidente la relación & & \\
\hline
\end{tabular}

Fuente: Datos obtenidos del cuestionario (2017); El Autor (2018).

Igualmente, el empleo de la Internet, cuya utilización se ve resentida por los problemas de conectividad en las escuelas, es una herramienta que debe utilizarse con mayor precisión en las orientaciones metodológicas para la búsqueda de documentos e información verdaderamente valiosa para el 
Proceso Enseñanza Aprendizaje (PEA) y sobre todo para que los estudiantes aprendan a discriminar la información valiosa de la superflua y desorientadora. El ejercicio crítico para por el desarrollo de una opinión clara y fundamentada de por qué asume un material y desecha otro.

Tabla 7. Indicador: Redes sociales

\begin{tabular}{|l|c|c|}
\hline \multicolumn{1}{|c|}{ Indicador: Redes sociales } & Selecciones & $\begin{array}{c}\% \text { del } \\
\text { total }\end{array}$ \\
\cline { 1 - 1 } No se aprecia & 1 & 3.84 \\
\cline { 1 - 1 } Se aprecia muy poco & 4 & 15.38 \\
\cline { 1 - 1 } Se aprecia & 21 & 80.76 \\
\cline { 1 - 2 } Se aprecia bien & 21 & \\
\cline { 1 - 2 } Es muy evidente la relación &
\end{tabular}

Fuente: Datos obtenidos del cuestionario (2017); El Autor (2018).

Aunque se presenta en un porcentaje alto (96.04\%) sin duda alguna tiene que ver con la actividad y orientación desde las asignaturas que dictan, no obstante, hay que tomar en consideración el poco desarrollo de propuestas coherentes, metodológicamente hablando, para el empleo de las redes sociales en el PEA de materias concretas.

Tabla 8. Indicador: Plataformas educativas (edmodo, moodle)

\begin{tabular}{|l|c|c|}
\hline \multicolumn{1}{|c|}{$\begin{array}{c}\text { Indicador: Plataformas educativas } \\
\text { (edmodo, moodle) }\end{array}$} & Selecciones & $\begin{array}{c}\% \text { del } \\
\text { total }\end{array}$ \\
\hline No se aprecia & 1 & 3.84 \\
\hline Se aprecia muy poco & 3 & 11.53 \\
\hline Se aprecia & 22 & 84.61 \\
\hline Se aprecia bien & & \\
\hline Es muy evidente la relación &
\end{tabular}

Fuente: Datos obtenidos del cuestionario (2017); El Autor (2018). 
Las plataformas educativas se emplean, pero pasan por el mismo problema de conectividad de internet y el desarrollo de la intranet en las escuelas, no obstante, se emplean y posibilitan, acorde al enfoque pedagógico de aprender a construir el conocimiento, ampliar y perfeccionar su empleo.

\section{Conclusiones}

Puesto que específicamente en la Educación Básica, el diseño curricular promueve la incorporación de las TIC en el proceso de enseñanzaaprendizaje, y por ende su democratización, se destaca y concluye que la escases o ausencia de centros informáticos y bibliotecas virtuales, así como poca o ninguna disponibilidad de software, equipos tecnológicos e Internet, aunado a la falta de dotación y accesibilidad de dispositivos técnicos, son los principales factores externos que no permiten la inserción definitiva de las TIC en la Educación Básica.

Concluyéndose además que como factores internos que influyen en el uso de las TIC por parte de los docentes de Educación Básica se destacan la baja formación en el área, la presencia de miedo y situaciones de estrés, así como apatía para afrontar circunstancias y condiciones de uso; reconocer que posiblemente el estudiante sepa y conozca más que el educador, quien debe estar ávido para adquirir nuevos conocimientos que le permitan manejar más y mejor el recurso informático, para poder ponerse a la par con sus alumnos.

Se puede concluir también que las TIC son herramientas novedosas al servicio de la educación, que van a proporcionan los medios y el nuevo contexto para producir un cambio en las formas de enseñar y aprender.

Por su parte los docentes opinan que las TIC ayudan a consolidar en la mayoría de los casos, los aprendizajes en la Educación Básica, mejorando la calidad de los procesos de enseñanza y aprendizaje, ya que con ellas se pueden abordar actividades más dinámicas y significativas, que producen un efecto positivo y deseable en los estudiantes, pues éstos pueden explorar y 
obtener otros conocimientos a su propio ritmo, mejorando el rendimiento escolar con el uso del computador.

Se agrega, como otra conclusión, que las TIC, facilitan el trabajo diario al poder realizar las planificaciones pedagógicas y las actividades, introduciendo estrategias digitales, que hacen a la función pedagógica más efectiva y eficiente; al respecto, se debe consideran la conformación de un equipo coordinado de trabajo, que se encargue de planificar la actualización de los docentes y la inclusión total de las TIC en la Educación Básica, así como velar que las salas de computación sean utilizadas por todos los docentes para la mejora de su enseñanza.

\section{Referencias}

Álvarez, S., Cuéllar, C., López, B., Adrada, C., Anguiano, R., Bueno, A., Comas, I., \& Gómez, S. (2011). Actitudes de los Profesores ante la Integración de las TIC en la Práctica Docente. Estudio de un grupo de la Universidad de Valladolid. Edutec-e. Revista Electrónica de Tecnología Educativa, (35), 1-19, ISSN: 1135-9250. Recuperado de: http://edutec.es/revista/index.php/edutec-e/article/download/416/152

Buela-Casal, G., \& Castro, A. (2009). Las Tecnologías de la Información y la Comunicación y la evaluación de la calidad en la Educación Superior. RUSC. Universities and Knowledge Society Journal, 6(2), 38, E-ISSN: 1698-580X. Recuperado de:

http://www.redalyc.org/articulo.oa?id=78012947007

Castro, S., Guzmán, B., \& Casado, D. (2007). Las TIC en los procesos de enseñanza y aprendizaje. Laurus, 13(23), 213-234, ISSN: 1315-883X. Recuperado de: http://www.redalyc.org/pdf/761/76102311.pdf

García, A. (2004). Calidad de la Educación en la Sociedad de la Información. Revista Complutense de Educación, 15(2), 509-520, ISSN: 1130-2496. Recuperado de: 
https://revistas.ucm.es/index.php/RCED/article/viewFile/RCED040422 $\underline{0509 A / 16175}$

Hüther, G. (2012). Biología del Miedo. El Estrés y los Sentimientos. Barcelona, España: Plataforma Editorial.

Marqués, P. (2006). Impacto de las TICs en Educación: Funciones y Limitaciones. Barcelona, España: Ediciones de Departamento de Pedagogía Aplicada de la Universidad Autónoma de Barcelona. [Documento en línea]. Recuperado de:

http://especializacion.una.edu.ve/iniciacion/paginas/marquestic.pdf

Mejía, A., Silva, C., Villarreal, C., Suarez, D., \& Villamizar, C. (2018). Estudio de los Factores de Resistencia al Cambio y Actitud hacia el Uso Educativo de las TIC por parte del Personal Docente. Boletín Virtual, 7(2), 53-63, ISSN: 2266-1536. Recuperado de:

http://revista.redipe.org/index.php/1/article/download/428/425/

Taylor, S., \& Bogdan, R. (2000). Introducción a los Métodos Cualitativos. Buenos Aires, Argentina: Ediciones Paidós. Recuperado de: https://asodea.files.wordpress.com/2009/09/taylor-s-j-bogdan-rmetodologia-cualitativa.pdf

Tramullas, J. (2002). Propuestas de Concepto y Definición de la Biblioteca Digital. III Jornadas de Bibliotecas Digitales JBIDI. San Lorenzo del Escorial. Madrid, España: Universidad Politécnica de Madrid. Recuperado de: http://eprints.rclis.org/15118/

Vaillant, D. (2014). Formación de Profesores en Escenarios TIC. Revista eCurriculum, 12(2), 1128-1142, ISSN: 1809-3876. Recuperado de: http://www.redalyc.org/pdf/766/76632206003.pdf 


\section{Oscar Antonio Martínez Molina \\ e-mail: oscar.martinez@unae.edu.ec}

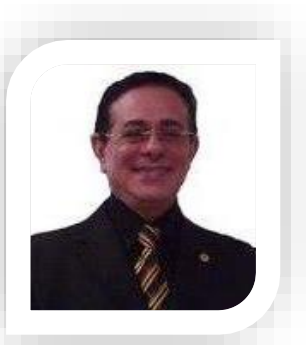

Nacido es San Cristóbal, estado Táchira, Venezuela. Residenciado en Cuenca, Ecuador. Licenciado en Educación Mención Orientación Educativa y Profesional (Universidad de Los Andes, Extensión Táchira). Magíster en Ciencias de la Educación Superior, Mención Andragogía (UNELLEZ). Doctor en Educación Mención Suma Cum Laude (Universidad de Málaga, España), Postdoctor en Estudios Libres de la Universidad Fermín Toro. Diplomado en Educación Abierta y a distancia (Universidad Fermín Toro). Maestría Experto avanzado en E-learning en la Fundación para la actualización tecnológica de Latinoamérica (FATLA). Maestría Experto en Tecnología Educativa nivel avanzado en la Fundación para la actualización tecnológica de Latinoamérica (FATLA). Profesor Jubilado de la Universidad Nacional Abierta. Categoría Académica de Titular. Director de tesis doctorales y de maestría. Responsable de la línea en enseñanza-aprendizaje de investigación del COBAIND. Representante del convenio Editorial Académica Española-COBAIND. Coordinador de la línea de investigación en Educación en la UNEFA Barinas. Presidente/Fundador del Consejo Barinés de Investigación y Desarrollo. Profesor investigador del Programa de Estímulo a la Innovación e Investigación en categoría "A-2". Docente investigación de la Universidad Nacional de Ecuador.

El contenido de este manuscrito se difunde bajo una Licencia de Creative Commons ReconocimientoNoComercial-Compartirlgual 4.0 Internacional 